\title{
MICROSTRUCTURE ANLAYSIS AND ENHANCEMENT OF NODULAR CAST IRON USING DIGITAL IMAGE PROCESSING METHOD
}

\author{
V.Sakthivel $^{1}$, P.Suresh $^{2}$, N.Mahalakshmi ${ }^{3}$ \\ ${ }^{1}$ Assistant Professor, Department of ECE, Paavai Engineering College, Namakkal District, Tamilnadu \\ ${ }^{2}$ Professor, Department of Mechanical Engineering, Karpagam College of Engineering, Coimbatore, Tamilnadu \\ ${ }^{3} P G$ Student, Department of ECE, Paavai Engineering College, Namakkal District, Tamilnadu
}

\begin{abstract}
Digital image processing is the technique to process pixel variations on digital images. This paper employs the digital image processing procedure for microstructure analysis and enhancement of nodular cast iron properties inorder to determine the quality factors. This processing made to sharpen and enhance the microstructure before quantitative analysis. This analysis taken in the analog form of nodular cast iron was performed manually such that, it is time consuming for multiple set of images. To avoid this criteria probably, Digital Image Processing techniques are being used. Often the nodular cast iron images are corrupted or defected during transmission by impulse noise which is often filtered using median filters. In this paper, some of processing steps as pre-processing, segmentation, filtering process and edge detection are done on these images for effective and lossless transmission ensuring the counting of nodules in microstructure and detailed study of grain properties that correlates with the mechanical properties such as ductility, malleability and brittleness. Only $30 \%$ restoration of image applicable to an acceptable level is limited. A new filtering method - Resolution Based Median Filter (RBMF) is proposed which achieves more than $95 \%$ restoration of nodular cast iron images to an acceptable level. This method optimizes further microstructure analysis of grain smoothening and grain boundary detection using finite element simulation model by varying the intensity level of images.
\end{abstract}

Keywords - Digital Image Processing, Material Microstructure, Edge Detection, Grain Boundary, Resolution Based Median Filter (RBMF).

\section{INTRODUCTION}

Digital image processing plays major role in wide range of applications. In such process, foremost field is processing and analysis of microstructure. It is the technique to process pixel variation on digital images using computer algorithms. It is more advantageous allowing complex algorithms to be implemented as simpler tasks and eliminates signal distortion and buildup noise during image processing. This digital processing can also be modeled for multidimensional systems by processing two dimensional images. One such application of digital image processing involves material processing of microstructure based nodular cast iron from imaging equipment.

The images are captured either by analog or digital camera. With varying properties such as noise parameter, lightening effect, resolution of camera, etc., digital image processing came into existence. It changes the poor image into good quality image. In this paper the images of nodular cast iron is taken and processed further for producing the meaningful results.

Microstructure analysis is defined as the structure of well organized surface of material which is obtained by a microscope of above $0.25 \mathrm{x}$ magnification. The microstructure of material is classified into metallic, polymeric, ceramic, and composite which influences the mechanical properties such as ductility, malleability, brittleness. The microstructure images captured either by analog or digital camera consist of superimposed noise usually impulse noise (salt and pepper noise) in the form of dark spots in the image. This occurs due to chemical reaction (micro inclusions) or due to some scratches in the image. The micro structural analysis is made on the nodular cast iron images which are often corrupted or defected while transmission by these impulse noises. The dark spots (salt and pepper noise) in the image are removed by using median filters.

For lossless and effective transmission, a fewer steps such as segmentation, pre-processing, edge detection and filtering process are done on these images. The first step includes segmentation of the image which predefines the threshold value by changing the level of intensity of the image. Next, the edge detection is done in order to detect the grain boundary of the nodular cast iron. This ensures the counting of nodules in microstructure and detailed study of grain properties that correlates with the mechanical properties. However the noise (dark spots) in the image of nodular cast iron is not completely removed. This paper mainly focuses on filtration techniques which gives a clear experimentation of nodular cast iron.

The previous papers based on median filter give restoration of about $30 \%$ and this limitation overcome by proposing a new method - Resolution Based Median Filter (RBMF) which restores more than $95 \%$ of the original image of 
nodular cast iron. Thereby, the image of nodular cast iron is enhanced. The rest of the paper comprises the following: Section II describes the theoretical background of digital image processing and nodular cast iron. Section III gives a detailed a study on segmentation, edge detection and methodology to process nodular cast iron. Section IV compares the median filters with the proposed method RBMF (Resolution Based Median Filter) and gives a detailed study on RBMF (Resolution Based Median Filter) algorithm. The experimental results and conclusion are discussed in Section VI.

\section{THEORETICAL BACKGROUND}

For the process of microstructure analysis, we have to study the theoretical background of digital image processing of nodular cast iron.

\subsection{Digital Image Processing}

The fig. 1 shows the generalized processing of an image. The input image $\mathrm{S}(\mathrm{x}, \mathrm{y})$ is taken in analog form using either by an analog or digital camera. The digitizer converts the input source signal to an electrical signal in which the amplitude of the image represents the pixel intensity of the image.

The converted electrical signal is digitized using an analog to digital converter usually in the form of two - dimensional signal $d(a 1, a 2)$. Then the digitized discrete signal is processed to obtain $\mathrm{p}(\mathrm{a} 1, \mathrm{a} 2)$. Finally the output image is displayed as S' $(x, y)$. The micro structural study on nodular cast iron specimen is prepared by the generalized digital processing system. The soft graphite phase is retained by sampling, grinding, polishing and etching with a suitable etchant in order to reveal the microstructure of cast iron.

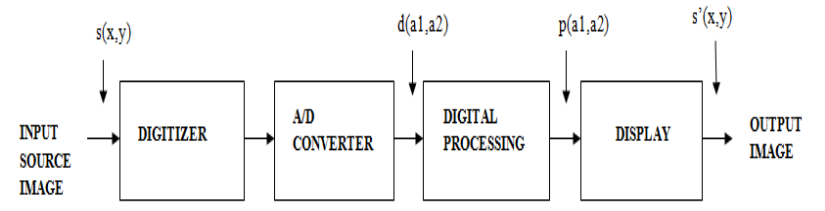

Fig 1: Generalized Image Processing System

\subsection{Nodular Cast Iron}

Nodular cast iron is also termed as the grey cast iron with their graphite particles are modified by magnesium added to the melt before casting. It consists of spheroid nodular graphite particles in ferrite. It differs from the grey cast iron with lamellar graphite in which its chemical composition is due to the basic elements which also contain very small quantities of magnesium (from 0.04 to $0.06 \%$ ), cerium and rare earths that influence the formation of graphite spheres. The properties of nodular cast iron are not only dependent upon the size, number and arrangement of graphite content in the cast iron, but also based upon the basic matrix consisting of ferrite and perlite. At the same hardness levels these nodular cast iron has much higher tensile strength than grey cast iron, therefore the toughness and elongation are great advantages of nodular cast iron._. As in case of grey cast iron, the tensile strength and strain define the quality of the cast iron.

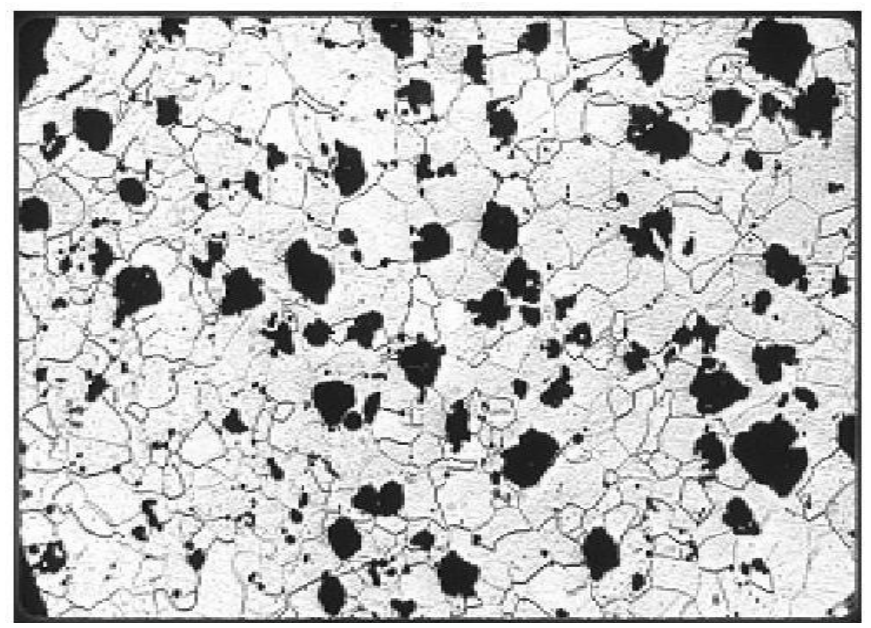

Fig 2: Nodular cast iron

\subsection{Need of Nodule Count}

The shape and structure of nodular cast iron and the counting of nodules present in the image results in the study of mechanical properties of nodular cast iron. The nodule count increases the metallurgical quality of the graphite. As the nodule count increases, the size and shape of nodular cast iron decreases which enhances the mechanical properties such as brittleness, malleability and ductility. Thus the need of nodule count enhances nodularity by increasing the nodule count.

\section{PROCESSING METHODOLOGY}

\subsection{Segmentation}

In digital image processing, image (nodular cast iron) segmentation is the process of partitioning a digital image into multiple segments (sets of pixels, also known as super pixels). The goal of segmentation is to simplify and/or change the representation of an image into meaningful image which is easier to analyze. It locates the objects and boundaries (lines, curves, etc.) in images and it assigns a label to every pixel in an image such that pixels with the same label share certain characteristics. The result of image segmentation is a set of segments that collectively cover the entire image, or a set of contours extracted from the image. The image segmentation is done by selecting a proper threshold value. This converts the gray-scale image into a binary image. The threshold method used in this paper is Ostu's method using global thresholding.

\subsection{Edge Detection}

The edge detection is an image processing technique for finding the boundaries of objects within images. It works by detecting discontinuities in brightness. It detects the edges in the image using matlab edge function as follows: 


$$
D-(D \oplus B)
$$

Where B represents the boundaries of the image in which binary logic ' 1 ' represents pixels of the image and binary logic ' 0 ' represents background of the image.

\subsection{Processing Steps}

The processing steps of micro structural analysis of nodular cast iron are as follows:

Soften the nodular cast iron with the small quantities of magnesium, cerium and rare earths to get the graphite spheres.

- $\quad$ Apply image segmentation to segment the image thereby preparing the specimen for metallurgical and mechanical properties examination.

- $\quad$ By pre-processing the segmented image, the noise is removed to a certain limit which occurs due to sampling, grinding, polishing and etching.

- $\quad$ The edge detection is performed to detect the grain boundaries of the image using various edge detection operators $\xi$.

- Count the region present in the micro structural image, using the above steps that correlate with the mechanical properties such as ductility, malleability, brittleness.

\section{RESOLUTION BASED MEDIAN FILTER (RBMF)}

The micro structural analysis is made on the nodular cast iron images which are often corrupted or defected while transmission by the impulse noises. By pre - processing the segmented image, the noise is removed to a certain limit which occurs due to sampling, grinding, polishing and etching. Using filtering techniques, the impulse noise present in the image will be suppressed and the corrupted or defected image can be restored to an acceptable level.

The impulse noise (salt and pepper noise) in the image of nodular cast iron is identified as dark spots which are removed by using median filters. The median filter preserves the sharp edges in the grain boundary region. The previous papers based on median filter give restoration of about only $30 \%$. This remains as the drawback of restoration of the original image. Hence a new method is proposed named - Resolution Based Median Filter (RBMF) which restores more than $95 \%$ of the original image of nodular cast iron. Thereby the image viewing of nodular cast iron is enhanced.

\subsection{RBMF Algorithm}

This algorithm primarily detects the impulse noise (dark spots) in the image. It identifies the corrupted and uncorrupted pixels in the image by proper window selection. The corrupted noisy pixels are replaced by noise free reliable pixel in the image. The detailed study on RBMF algorithm is as follows:
Step: 1 - Initialize the first pass, $X=1$ starting from $n_{1}=1$ and $\mathrm{n}_{2}=1$ and move forward direction.

Step: 2 - Set the window size, $\mathrm{K}$ and set of uncorrupted pixels $\xi$ i.e. $\mathrm{K}==3 ; \xi=\theta$.

Step: 3 - The square window $\mathrm{K} \times \mathrm{K}$ have a set of pixel centered at $\mathrm{n}=(\mathrm{n} 1, \mathrm{n} 2)$ is defined as,

$$
\begin{gathered}
\alpha_{n}^{k}=\left\{l=\frac{l_{1} l_{2}}{n_{1}}-\frac{k-1}{2} \leq l_{1} \leq n_{1}+\frac{k-1}{2}, n_{2}-\frac{k-1}{2} \leq l_{2} \leq\right. \\
\left.n_{2}+\frac{k-1}{2}\right\}
\end{gathered}
$$

Where $\mathrm{K}$ is an odd integer not less than 3 which indicates the size of the local neighborhood. The phase begins by analyzing the pixel-wise characteristics of the corrupted image, $\mathrm{Y}$ in the local neighborhood, $\mathrm{K} x \mathrm{~K}$.

Step: 4 - If $F_{1}<Y_{n}<F_{2}$ then $Y_{n}$ is declared uncorrupted and so the pixel is retained in the corresponding pixel position of the restored image, $\mathrm{V}$

$$
\begin{aligned}
& \text { i.e } \quad V_{n}^{X}=Y_{n} \\
& F_{1}=\operatorname{minimum}\left\{Y_{l} / l \in \alpha_{n}^{k}\right. \\
& F_{2}=\operatorname{maximum}\left\{Y_{l} / l \in \alpha_{n}^{k}\right.
\end{aligned}
$$

Subsequently the flag image at position $\mathrm{n}, z_{n}$ is reset to ' 0 ' indicating a non-impulsive position. Now the algorithm is continued from Step 9.

Step: 5 - The purity status of Yi cannot be concluded corrupted when $\mathrm{Yi}$ is found not to lie strictly in between F1 and F2. The uncorrupted pixels in the neighborhood around $\mathrm{Y} \mathrm{i}$ are caught in the impulse free pixel set, $\xi$ i.e

$$
\xi=\left\{\frac{\mathrm{Y}_{\mathrm{l}}}{\mathrm{l}} \in \alpha_{\mathrm{n}}^{\mathrm{k}} \text { and } \mathrm{F}_{1}<\mathrm{Y}_{\mathrm{l}}<\mathrm{Y}_{2}\right\}
$$

Step: 6 - If the pixel is found corrupted and $\xi$ being non empty then $Y_{n}$ is once again checked for its purity by analyzing it's correlation with the uncorrupted pixels of the window, $\mathrm{K} \times \mathrm{K}$ for which $\xi_{\min }$ and $\xi_{\max }$ is determined in such a way that

$$
\xi \min =\min (\xi) ; \xi \max =\max (\xi)
$$

The Minimum Deviation (MD) of yi with $\xi$ min and $\xi \max$ is determined which is defined by

$$
\mathrm{MD}=\operatorname{minimum}\{\xi \min -\mathrm{Yi}, \xi \max -\mathrm{Yi}\}
$$

Step: $7-$ Here if $\xi \neq 0$ and $\mathrm{MD} \leq \mathrm{Th}$, the pixel $\mathrm{Yi}$ is declared uncorrupted and is maintained. Since it's correlation with the nearest uncorrupted signals is high. The corresponding flag image is also updated to ' 0 '. Now the algorithm is continued from Step 9. 
Step: 8 - otherwise if $\xi \neq 0$ and MD > Th, then the pixel $\mathrm{Y}_{\mathrm{i}}$ is replaced by the median of the pixels in $\xi$ which is the set of uncorrupted pixels in the nearest possible neighborhood of $\mathrm{Y}_{\mathrm{i}}$. Thus the restored output at the position $\mathrm{i}$ in the filtered image, $\mathrm{V}$ is obtained as follows.

$$
V_{n}^{X}=\operatorname{medium}(\xi)
$$

Subsequently the flag image yi is reset to ' 0 ' indicating a non-impulsive position since we replaced the corrupted pixel in the restored image. If $\xi$ is found empty at this stage, $Y_{i}$ is replaced by the mean of four neighbors of the previously processed image as in $V_{n}^{X}$ (refer at the bottom of the page).

Step: 9 - Move to next pixel for processing and repeat Step 1 through Step 8.

$$
\begin{aligned}
V_{n}^{X}= & \left\{\left(\frac{1}{4}\right) *(U x(n 1-1, n 2-1)+U x(n 1-1, n 2)+U x(n 1-1, n 2+1)+U x(n 1, n 2-1)) \text { if } X=1\right. \\
& \left(\frac{1}{4}\right) *(U x(n 1+1, n 2+1)+U x(n 1+1, n 2)+U x(n 1+1, n 2-1)+U x(n 1, n 2+1) \text { if } X=2 \\
& \left(\frac{1}{4}\right) *(U x(n 1+1, n 2+1)+U x(n 1+1, n 2)+U x(n 1+1, n 2-1)+U x(n 1, n 2-1) \text { if } X=3 \\
& \left(\frac{1}{4}\right) *(U x(n 1-1, n 2+1)+U x(n 1-1, n 2)+U x(n 1-1, n 2-1)+U x(n 1, n 2+1) \text { if } X=4
\end{aligned}
$$

Step: 10 - Increment $X=X+1$ Move to Step 2 and process each pixel in the reverse passion from $\mathrm{n} 1=\mathrm{s}$ and $\mathrm{n} 2=\mathrm{p}$.

Step: 11 - Increment $X=X+1$ and Move to Step 2 and process each pixel in the bottom left to top right passion from $\mathrm{n} 1=\mathrm{s}$ and $\mathrm{n} 2=1$ from $\mathrm{n} 1=\mathrm{s}$ and $\mathrm{n} 2=\mathrm{p}$.

Step: 12 - Increment $\mathrm{X}=\mathrm{X}+1$ and Move to Step 2 and process each pixel in the reverse passion from $\mathrm{n} 1=1$ and $\mathrm{n} 2=\mathrm{p}$ from $\mathrm{n} 1=\mathrm{s}$ and $\mathrm{n} 2=\mathrm{p}$.

Step: 13 - Once the above four passes are over, we replace all the pixels in the final restored image $U$ by the median of the 4 individual pixel values in the above four scanning procedure.

\section{EXPERIMENTAL RESULTS}

Using thresholding and various edge detection operators, the grain boundary of the image captured is detected. The results are shown below:

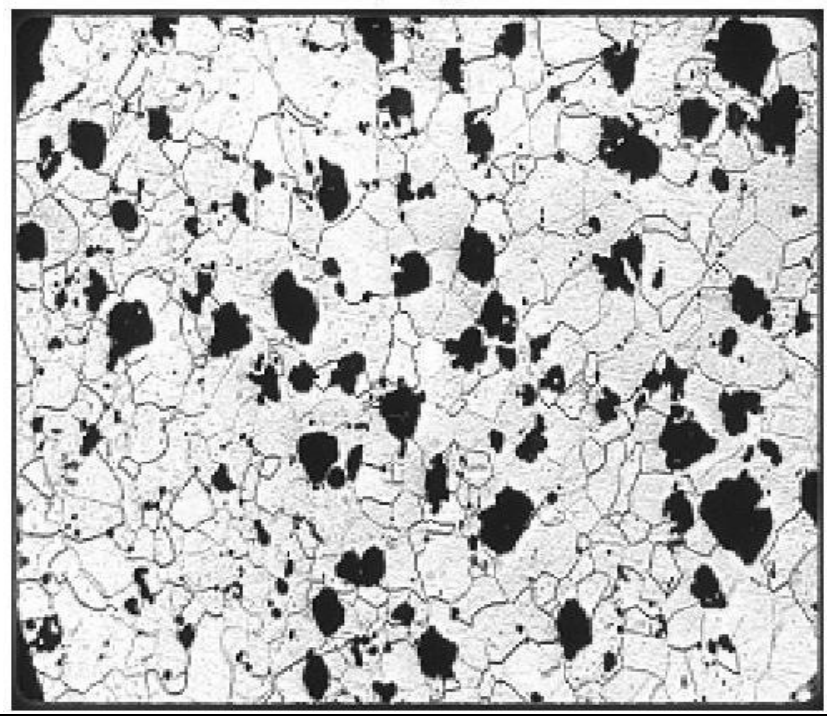

Fig 3: Captured Image

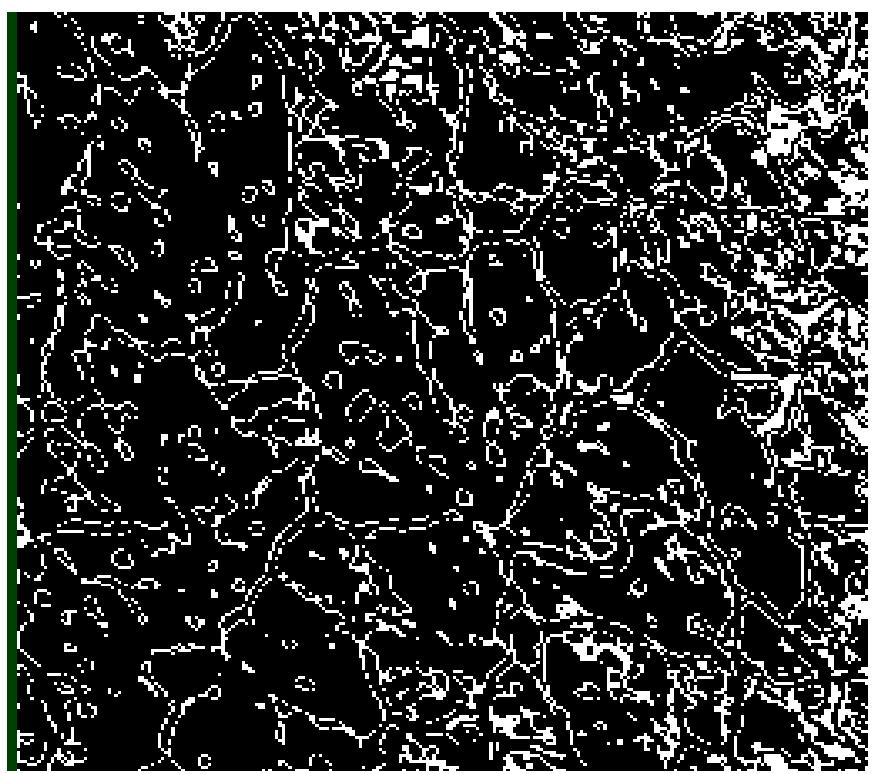

Fig 4: Prewitt's Edge Detection Operator

Prewitt edge detection calculates the maximum response of a set of convolution kernels to find the local edge orientation for each pixel . 


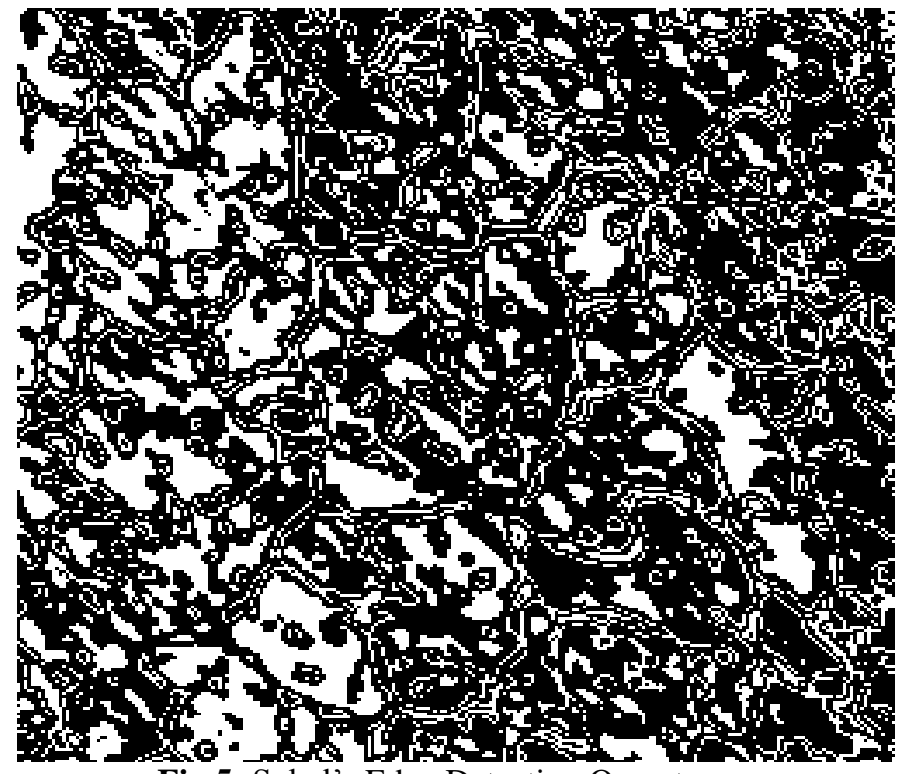

Fig 5: Sobel's Edge Detection Operator

Sobel's edge detector provides better smoothing (noise suppression) of the image.

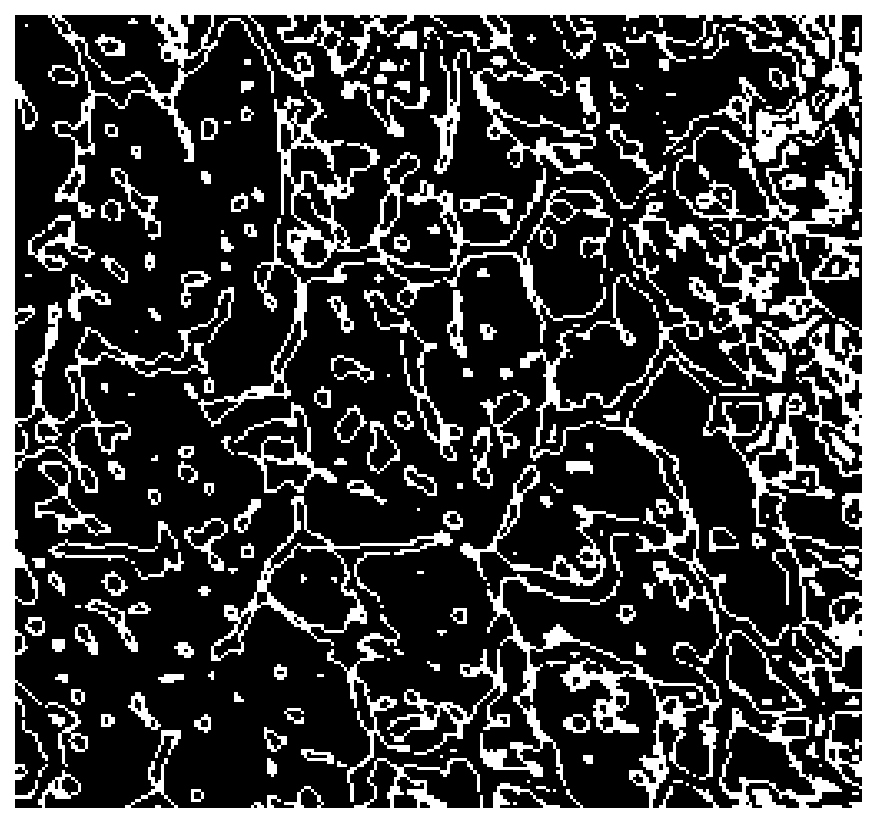

Fig 6: Robert's Edge Detection Operator

Robert's edge detector calculates the square root of the magnitude squared of the convolution with the Robert's row and column edge detectors.

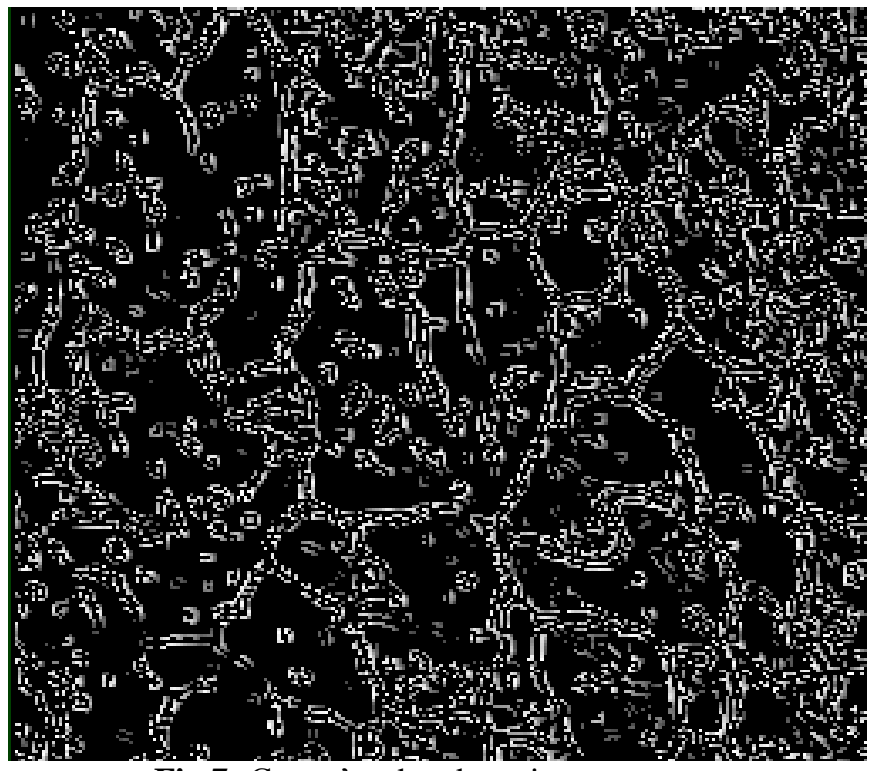

Fig 7: Canny's edge detection operator

The Canny's edge detector smoothens the image and then finds the image gradient to highlight regions with high spatial derivatives.

The above results count the number of nodules by varying the intensity level of the image. Hence the grain boundary is detected and smoothening of the image is done in the microstructure.

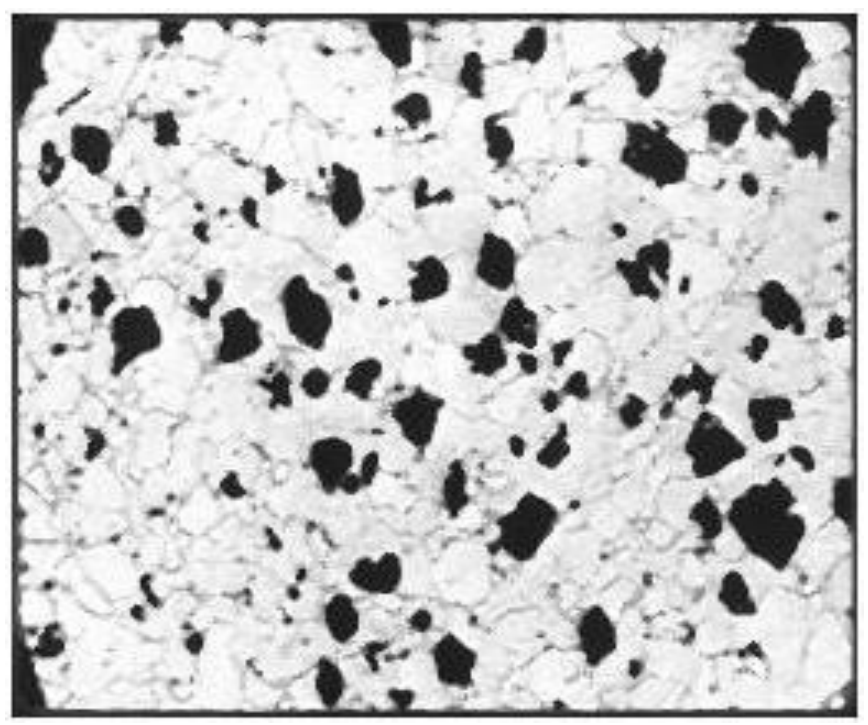

Fig 8: Median Filter Output

\section{CONCLUSION}

The paper discusses about the new algorithm method RBMF and results of the various edge detection operator. The experimental research for implementing $\mathrm{RBMF}$ is in progress. The median filtering has been used to remove the impulse noise in the captured nodular cast iron and the grain boundary has been detected using different edge detection operator. The mechanical properties such as ductility, malleability, brittleness are achieved by counting the number of nodules in the image. 


\section{REFERENCES}

[1]. H.Sarojadevi, Ambikashri B. Shetty, Apoorva K. Murthy, P. Balachandra Shetty, Dr.P.G Mukunda, "Digital Image Processing Technique for Microstructure Analysis of Spheroidal Graphite Iron" on International Journal of Combined Research \& Development (IJCRD), Volume: 1; Issue: 4; August -2013 .

[2]. Geoffrine Judith.M.C and N.Kumarasabapathy"Study And Analysis Of Impulse Noise Reduction Filters" on Signal \& Image Processing: An International Journal (SIPIJ) Vol.2, No.1, and March 2011.

[3]. S.G. Lee, Y. Mao, A.M. Gokhale, J. Harris, and M.F.Horstemeyer (2009), Application of digital image processing for automatic detection and characterization of cracked constituent particles/inclusions in wrought aluminum alloys, Elsevier, Materials Characterization, 60(9), pp.964-970.

[4]. D. Deb, S. Hariharan, U.M. Rao, and Chang-Ha Ryu (2008), Automatic detection and analysis of discontinuity geometry of rock mass from digital images, Elsevier, Computers \& Geosciences, 34(2), pp.115-126.

[5]. Zhanfeng Shen, Jiancheng Luo, Chenghu Zhou, Guangyu Huang, Weifeng Ma, and Dongping Ming (2005), System design and implementation of digital-image processing using computational grids, Elsevier, Computers \& Geosciences, 31(5), pp. 619-630.

[6]. D.W.Moolman, C. Aldrich, J.S.J. Van Deventer, and W.W. Stange (1994), Digital Image Processing as a tool for online monitoring of froth in flotation plants, Elsevier, Minerals Engineering, 7(9), pp. 1149-1164.

[7]. S.Vandenberg and C. F. Osborne (1992), Digital image processing techniques, fractal dimensionality and scalespace applied to surface roughness, Elsevier, Wear, 159(1), pp.17-30. 